\title{
Does "Yummy" Food Help You Grow and Avoid Illness? Children's and Adults' Understanding of the Effect of Psychobiological Labels on Growth and Illness
}

\author{
Lakshmi Raman \\ Department of Psychology, Oakland University, Rochester, MI 48309, USA \\ Correspondence should be addressed to Lakshmi Raman, raman@oakland.edu
}

Received 5 March 2011; Revised 16 May 2011; Accepted 15 June 2011

Academic Editor: Susan A. Gelman

Copyright () 2011 Lakshmi Raman. This is an open access article distributed under the Creative Commons Attribution License, which permits unrestricted use, distribution, and reproduction in any medium, provided the original work is properly cited.

\begin{abstract}
Three studies examined children's understanding of the role of psychobiological labels such as tasty ("yummy") and not tasty ("yucky") foods on growth and illness. Studies 1 and 3 examined the role of tasty and not tasty foods on height, weight, and illness, respectively. Study 2 controlled for the possibility that participants were responding to the positive and negative valence of the terms "yummy" and "yucky" in Study 1. Results revealed that young children entertain psychobiological causes for growth but not for illness. These results suggest that young children selectively apply psychobiological causes to explain different biological processes.
\end{abstract}

\section{Introduction}

Research on children's understanding of biological processes has primarily focused on children's causal recognition of illness, physical development, and growth. The results of most of these studies have demonstrated that children as young as 3 years of age consistently employ biological causes for biological processes and that they reject nonbiological causes such as immanent justice (Backsheider et al. [1]; Inagaki [2]; Kalish [3]; Raman and Gelman [4]; Springer and Keil [5]; Springer and Ruckel [6]).

However, research on children's recognition of the impact of psychological factors on biological processes has generated mixed results. Some studies have demonstrated that young children and even adults entertain psychological factors for the origins of common contagious illness (Raman and Gelman [7]; Raman [8]; Schulz et al. [9]), whereas other studies have found that young children entertain biological causes for dual causal biological processes such as psychogenic illnesses (Notaro et al. [10]). One of the major limitations of these studies is that they have all primarily focused only on the biological process of illness.

The following studies will further clarify this debate by examining another subdomain of biology that is of extreme importance but one that has received minimal attentionchildren's understanding of the role of psychobiological factors. Psychobiological factors are factors that present an interaction of biological causes and psychological causes. There have been no studies that have examined the impact of an interaction of biological and psychological causes on biological processes, but there is a plethora of studies that have examined the role of psychological factors on the contraction of common contagious illnesses. The results of these studies have presented mixed results. Some studies have demonstrated that preschoolers maintain a strict demarcation between the psychological and biological domains. For example, Kalish [3] demonstrated that preschoolers recognize that knowledge determines mental reactions to contamination but physical contact determines bodily reactions. Notaro et al. [10] found that, in the area of bodily responses such as headaches and stomach aches, preschoolers tended to view these as purely physical processes. However, there have been other studies that have demonstrated that preschoolers entertain psychological factors for biological processes. Inagaki [11] and Inagaki and Hatano [12] found that while the majority of Japanese 4-6-year old reasoned that biological activities would affect the susceptibility to catching a cold, these children also believed that social/psychological factors 
(such as telling a lie or pinching a friend) would results in catching an illness. Raman and Gelman [7] found that preschoolers and first and second graders reasoned that people were more likely to contract a contagious illness from someone they did not know than someone they knew suggesting that social relatedness can influence the contraction of an illness.

The above studies have examined the effect of psychological and social factors. However, the present studies examine the interaction of biological and psychological factors on growth and illness. One of the factors that make the study of psychobiological factors interesting is that the hedonic and healthy evaluations of many foods are usually highly correlated, thus making it difficult to disentangle the two.

In "yummy" (tasty) and "yucky" (not tasty) nutrition, the "yummy" and "yucky" components are the psychological components since taste is subjective and the healthy/ unhealthy nutrition is the biological component. Thus, the effect of "yummy" or "yucky" healthy/unhealthy foods would be assessing the impact of psychobiological factors on the biological process of growth and illness.

One pressing question is what makes the impact of nutrition on growth and illness an interesting one to study? Nutrition is an interesting biological causal factor as it can have both positive and negative biological consequences that can be consciously controlled via the kind of food eaten. Healthy nutrition can have positive biological effects such as contributing to height but reducing weight and susceptibility to illness whereas unhealthy nutrition can have the opposite effect. Thus, unlike other biological agents that usually have only one type of consequence (e.g., germs that cause illness), nutrition can have dual consequences depending on the context. Growth is a biological process that reflects involuntary normal development whereas illness is a deviant biological process that deters normal development. Most growth is irreversible whereas most illnesses are reversible. Growth has clear causes and explanations; illness is a phenomenon that can arise out of nowhere with the causal underpinnings of illness not always clear. Similarly for the process of illness, consuming unhealthy foods can make an individual more susceptible to contract an illness whereas consuming healthy foods can have the opposite effect. Thus, the fact that there is no straightforward cause and effect relationship of the effects of nutrition on growth and illness makes this an interesting topic to study.

However, even within the domain of nutrition, there have been few studies that have examined American children's understanding of nutrition on the biological process of growth and, currently, there are no studies that have examined the impact of nutrition on contracting an illness in Western populations. Wellman and Johnson [13] examined what children know about a variety of nutritional inputs and the consequences of different diets. Although they found that, even kindergartners know that some diets lead to growth and health whereas other diets lead to weight gain and laziness, kindergartners differed from third and sixth graders in that they thought that consumption of any food would lead to a gain in height and weight. Contento [14] documented that preschoolers have some idea of input-output relations with many misconceptions but they virtually have no model as to what mediates these relationships. Nguyen [15] found that by age 4, children selectively could infer that healthy and junky foods have an effect on the human body (Cheetos made Jake's body daxy) but not on materials/substances or unrelated inferences. The finding also demonstrates that children can extend the effect of one food on the human body in one category to other foods in the same category. Slaughter and Ting [16] found that between the ages of 5 and 8 years Australian children demonstrated a significant increase in mechanistic and vitalistic reasoning about food and nutrition when assessing the purpose of eating, effects of different quantities of food, effects of specific foods, and effects of an unbalanced diet. In a non-Western population, Inagaki [2] found that Japanese 6 -year olds recognized that nutrition could influence the susceptibility to illness and that 6-year olds could generate some explanations as to why this was the case.

Other studies have examined children's conceptual understanding of what makes food healthy/unhealthy. McKinley et al. [17] found that older children classified food based on nutritional value as opposed to younger children who based their judgments on the taste and enjoyment of the food. However, Guerin and Thibaut [18] found that even 6year olds classified fruits as healthy and that children don't always have a preference toward fun foods. Nguyen [15] found that young children based their responses on how foods would impact health and growth when classifying foods as healthy or junky. Thus, the studies that have emerged in the domain of nutrition have focused on children's ability to classify foods and the impact of these foods on growth. There have been no studies that have examined the role of psychobiological factors on the biological process of growth and illness.

The following three studies make a novel contribution to the literature on children's understanding in the following ways: (a) the studies are the first of their kind to examine the interaction of the role of psychobiological factors (yummy and yucky healthy foods, and yummy and yucky unhealthy foods) on growth and illness. Raman [19] found that even preschoolers entertained the notion that the type of food eaten influences mood states. Thus, it would be interesting to combine the biological and psychological factors to determine if children would subscribe to the notion of psychobiological labels affecting biological processes; (b) these studies are the first to examine the impact of the psychological perception of a biological agent (nutrition) simultaneously on two biological processes-growth (height and weight) and illness; (c) finally, these studies are the first to examine what kind of underlying mechanisms children entertain for the causal processes of growth and illness.

Study 1 examines preschoolers through adults' perceptions of the role of consuming "yummy" and "yucky" healthy and unhealthy foods on height and weight. Study 2 controls for positive and negative valence factors that might have been contributing to the developmental differences that were obtained in Study 1. Study 3 examines the role of "yummy" and "yucky" healthy and unhealthy foods on the probability of contracting common contagious illnesses. 
The following hypotheses are proposed: (a) if children entertain only biological causes for biological processes, they should not think that psychological labels such as the "yumminess" or the "yuckiness" of a food will affect biological processes; (b) however, if children think that psychobiological labels affect growth and/or illness, we might see evidence of a global or a differential effect of "yummy" and "yucky" nutrition on promoting growth and illness.

\section{Study 1}

In Study 1, the major question of interest is whether children and adults think that psychobiological factors such as "yummy" and "yucky" nutrition affect the two components of growth-height and weight. The causal factor of nutrition often has opposing effects on height and weight. For example, healthy nutrition usually promotes an increase in height but not an increase in weight. In fact, adopting a diet that consists primarily of healthy foods often promotes a decrease in weight whereas adopting a diet of unhealthy foods would promote an increase in weight but not in height. Raman [19] found that preschoolers and second graders reasoned that overall nutrition contributes to increased height and weight, but it was only at fourth grade that children recognized the differential effects of healthy and unhealthy nutrition. However, even preschoolers judged that the type of nutrition affects psychological mood states, with healthy eaters being in a positive mood state and unhealthy eaters being in a negative mood state. This demonstrates that young children may have an earlier recognition of a mind-body interaction than they do of the biological causal processes involved in growth. Thus, it would be interesting to determine if young children entertain affective factors such as "yummy" and "yucky" as overriding the nutritional value of the food in question. Based on previous studies (Notaro et al. [10, 20]; Raman, [7]; Schultz et al. [9]), two possibilities might emerge. The first possibility is that children might clearly not entertain psychobiological factors as affecting height and weight. The second possibility is that children might entertain the notion that psychobiological factors can affect height and/or weight.

\subsection{Method}

\subsubsection{Participants}

Pretest. Prior to this study, a separate group of adults $(n=$ $30)$ and children $(n=30)$ were presented with a list of healthy and unhealthy foods and asked to rate them as "yummy" or "yucky." The items most frequently listed in each category were selected as representative of healthy and unhealthy foods. In this way, a variety of healthy and unhealthy foods were presented so that the food groups were not narrowly defined by what the experimenter viewed as yummy/yucky healthy and unhealthy foods. The twelve most frequently mentioned "yummy" foods and the twelve most frequently mentioned "yucky" foods across both age groups were then selected to be included in the study. The common
TABLE 1: Selection of healthy and unhealthy foods presented for Studies 1-2.

\begin{tabular}{lc}
\hline Healthy foods & Unhealthy foods \\
\hline Broccoli & Hash browns \\
Grapes & Potato chips \\
Peas & Donuts \\
Apples & Candy \\
Cereal & French fries \\
Oatmeal & Jelly beans \\
\hline
\end{tabular}

features of the healthy foods were that they were all low in fat and high in nutritional value whereas the unhealthy foods were all high in fat and sugar (see Table 1 for a complete list of healthy and unhealthy foods).

Main study. The main study consisted of 25 preschoolers (12 females and 13 males, $M$ age $=4$ years 5 months, range $=4$ years to 4 years 11 months ), 27 second graders (12 females and 15 males, $M$ age $=7$ years 6 months, range $=7$ years to 8 years 3 months), 24 fourth graders (10 females and 14 males, $M$ age $=9$ years 4 months, range $=9$ years to 10 years 1 month), 23 sixth graders (10 females and 13 males, $M$ age $=11$ years 5 months, range $=11$ years 1 month to 12 years 3 months), and 33 adults (17 females and 16 males, $M$ age $=19$ years 5 months, range $=18$ years to 23 years and 3 months). Participants were residents of a small midwestern city. Children were recruited from preschools in the area. Adults were recruited from an introductory psychology pool at a state university and were given course credit for their participation.

Main Task. The main task presented each participant with two conditions, height and weight. Within each condition, each participant was presented with 12 vignettes (6 describing characters who consumed healthy foods and 6 describing characters who consumed unhealthy foods), resulting in each participant being presented with a total of 24 vignettes.

The questions for the healthy and unhealthy foods were identical except for the foods listed in each vignette. An example for one of the vignettes for healthy foods is "There are two boys, Jake and Oliver. Both Jake and Oliver eat the same amount of peas for lunch. Jake thinks that peas are a "yummy" food. Oliver thinks peas are a "yucky" food." To ensure that the youngest children retained this information, two memory questions were asked: (a) who thinks peas is a "yummy" food? who thinks peas is a "yucky" food? Now remember that Jake ate all of his peas for lunch and Jake thinks that peas are a "yummy" food. Oliver ate all of his peas for lunch and Oliver thinks that peas are a "yucky" food. Who do you think is going to grow taller? (a) Jake; (b) Oliver; (c) they will both grow the same. Why do you think (child's response) is going to grow taller? Is it because (a) yummy/yucky food will make you grow tall (psychological justification); (b) yummy/yucky foods have more nutrients in them which will make you grow tall (psychobiological justification); (c) peas have a lot of nutrients in them and 
the nutrients will make you grow tall (biological justification). Is there any other reason why (child's response) is more likely to grow taller? If the child chooses "same" response, they are asked "Why do you think they will both grow the same?" Is it because: (a) peas have a lot of nutrients in them and the nutrients will make you grow taller (biological justification); (b) the yumminess/yuckiness of a food does not matter; they are both eating the same amount of peas (nonbiological justification); (c) yummy/yucky food will make you grow taller (psychological justification). Finally, participants were asked if they thought there was any other reason that (child's response) would grow taller or grow the same, depending on the response of the participant. To reduce cognitive demands on the younger participants, justifications were asked for 3 of the healthy foods and 3 of the unhealthy foods for the tall condition, and 3 of the healthy foods and 3 of the unhealthy foods for the fat condition. The items for which justifications were generated for the tall condition were different from items for which justifications were generated for the fat condition. Thus, across each participant, justifications were generated for a total of 12 foods across both conditions. Items were randomized within each condition and the response options and conditions were counterbalanced across participants such that half of the participants received the height condition first and the other half received the weight condition first.

2.2. Procedure. Children were interviewed individually. They were shown hand-drawn black-and-white stick figure drawings of the two characters in each question. The purpose of using pictures was to hold the child's attention; the pictures did not make reference to height or weight. The experimenter read the vignettes to the children, pointing to the relevant character when reading the vignettes. Participants were asked to answer each vignette, and their responses were noted. College students received a testing booklet that contained 24 items (with no pictures). They were asked to read the vignettes and write down their responses in the booklets.

\subsubsection{Results}

Coding of Judgments. Judgments were coded in three different ways to determine how many "yummy" responses, how many "yucky" responses, and how many "same" responses participants gave. For the "yummy" analysis, if participants chose the character who said that the food was "yummy," this was coded " 1 ," all other responses were coded " 0 ." For the "yucky" analysis, if participants chose the character who said that the food was "yucky", this was coded " 1 " and all other responses were coded "0." For the "same" analysis, if participants chose the "same" response, this was coded " 1 " and all other responses were coded " 0 ." Thus, for each participant for each of the three categories_- "yummy," "yucky," and "same," there were four totals in each-yummy responses for healthy foods in the tall and fat categories and yucky responses for unhealthy foods in the tall and fat categories. A descriptive analyses of the data revealed that the majority of the participants chose either the "yummy" or
TABle 2: (a) Mean number of "yummy" responses (out of a maximum of 6). (b) Mean number of "yucky" responses (out of a maximum of 6). (c) Mean number of "same" responses (out of a maximum of 6 ).

(a)

\begin{tabular}{lcccc}
\hline \multirow{2}{*}{ Age group } & \multicolumn{2}{c}{ Tall } & \multicolumn{2}{c}{ Fat } \\
& Healthy & Unhealthy & Healthy & Unhealthy \\
\hline Preschool & 4.4 & 3.9 & 4.4 & 4.2 \\
Second & 3.1 & 1.8 & 2.0 & 3.0 \\
Fourth & 1.0 & 0.2 & 0.2 & 0.9 \\
Sixth & 1.7 & 0.2 & 0.2 & 2.3 \\
Adults & 0.4 & 0.1 & 0.1 & 0.9 \\
\hline
\end{tabular}

(b)

\begin{tabular}{lcccc}
\hline \multirow{2}{*}{ Age group } & \multicolumn{2}{c}{ Tall } & \multicolumn{2}{c}{ Fat } \\
& Healthy & Unhealthy & Healthy & Unhealthy \\
\hline Preschool & 0.3 & 0.6 & 0.6 & 0.5 \\
Second & 0.1 & 0.8 & 1.4 & 0.0 \\
Fourth & 0.0 & 0.0 & 1.3 & 0.4 \\
Sixth & 0.0 & 1.9 & 0.6 & 0.1 \\
Adults & 0.0 & 0.2 & 0.8 & 0.2 \\
\hline
\end{tabular}

(c)

\begin{tabular}{lcccc}
\hline \multirow{2}{*}{ Age group } & \multicolumn{2}{c}{ Tall } & \multicolumn{2}{c}{ Fat } \\
& Healthy & Unhealthy & Healthy & Unhealthy \\
\hline Preschool & 1.3 & 1.5 & 1.0 & 1.4 \\
Second & 2.9 & 3.3 & 2.7 & 3.0 \\
Fourth & 4.9 & 4.9 & 5.4 & 5.1 \\
Sixth & 4.3 & 3.8 & 4.5 & 3.3 \\
Adults & 5.5 & 5.7 & 5.3 & 5.0 \\
\hline
\end{tabular}

the "same" responses across all categories; thus, the focus of the analyses will be on these two categories (see Tables 2(a), 2(b), and 2(c) for means across age groups for "yummy," "yucky" and "same" responses).

A "yummy" analysis was conducted to determine if participants thought that "yummy" healthy and unhealthy foods would have an effect on height and weight. A 5 (age group) $\times 2$ (food type) $\times 2$ (condition) analysis of variance (ANOVA) was conducted with a focus on the influence of "yummy" labels on height and weight. The results revealed a condition $\times$ food interaction, $F(1,125)=39.76, P<.01$, a condition $\times$ food $\times$ age group interaction, $F(4,125)=37.87$, $P<.01$, and an age group effect, $F(4,125)=32.8, P<.01$. Post hoc Bonferroni-corrected $t$-tests revealed that, overall, participants thought that "yummy" healthy foods are more likely to lead to an increase in height, $P$ s $<.01$, whereas "yummy" unhealthy foods are more likely to lead to an increase in weight, $P<.01$. Second graders, fourth graders, and sixth graders reasoned that healthy "yummy" food would result in an increase in height, $P$ s $<.05$. However, only second graders, sixth graders, and college students reasoned that unhealthy "yummy" food would result in an increase in weight, $P s<.01$. The triple interaction revealed that 
preschoolers thought that "yummy" healthy foods would result in a significant increase in height compared to all other age groups, $P s<.01$. Sixth graders generated a greater number of "yummy" responses for healthy foods for height, compared to college students, $P<.03$. For unhealthy foods, preschoolers and second graders gave a greater number of "yummy" responses than the other age groups, $P$ s $<.01$. For weight, preschoolers gave more "yummy" responses for healthy foods than the other age groups, $P s<.01$. For unhealthy foods, preschoolers gave more "yummy" responses compared to fourth graders, sixth graders, and college students, $P$ s $<.01$. A "same" response analysis revealed a food $\times$ age group interaction, $F(4,125)=$ $5.29, P<.01$ and an age group effect, $F(4,125)=21.67$, $P<.01$. Post-hoc Bonferroni-corrected $t$-tests revealed that, for healthy foods, preschoolers and second graders gave significantly less of the "same" responses compared to other age groups, $P<.02$. Sixth graders were the only participants who showed more of the "same" responses for healthy foods than unhealthy foods for growth, $P<.01$.

Coding of the Justifications. Justifications were analyzed for the "yummy," "yucky," and "same" responses in the following way: for the "yummy" responses, if participants chose the character who said "yummy" and selected the psychological justification, it was coded as " 1 ," the biological justification it was coded as "0", and the psychobiological justification was coded "0.5." For the "yucky" responses, if participants chose the character who said "yucky" and selected the psychological justification, it was coded "1," the biological justification was coded " 0 " and the psychobiological justification was coded "0.5." For the "same" responses, if participants chose the character who said "same" and gave a biological justification, it was coded " 1 ," the nonbiological quantity justification was coded "0.5." The open-ended responses were coded independently by two separate coders and the kappa was 0.9 .

Analyses of the Justifications. For the "yummy" responses, $91 \%$ of preschoolers, $18 \%$ of second graders, $56 \%$ of fourth graders, $77 \%$ of sixth graders, and $38 \%$ of college students gave psychological justifications. Second graders primarily gave biological justifications (83\%). For the "same" responses, non-biological quantity responses were the most prominent with $94 \%$ of preschoolers, $99 \%$ of second graders, $97 \%$ of fourth graders, $84 \%$ of sixth graders, and $93 \%$ of college students selecting the quantity justification

2.3. Discussion. This study examined the impact of psychobiological factors such as "yummy" and "yucky" foods on growth. The most interesting result of this study was the developmental difference obtained between younger and older children in their reasoning about the effect of psychobiological factors on growth. Although the number of "yummy" judgments decreased sharply after second grade, analyses of the judgments revealed that when fourth and sixth graders chose the "yummy" response, they justified their choices by opting for the psychological justification.
This demonstrates that their justifications were in line with their forced choice judgments.

One surprising result is the lack of biological justifications across all age groups when participants chose the "same" response. Instead, across all age groups, when participants chose the "same" response, they opted for the non-biological quantity response- "they both ate the same amount of (the food in question) so they will both grow the same" rather than "(the food in question) has nutrients in it so they will both grow the same." The focus seemed to be on the external attributes of the food (the quantity) rather than the underlying nutritional attributes.

The results of this study demonstrate that young children think that psychological factors such as the "yumminess" of a food will have an effect on the impact of the food eaten. This suggests that the biological and psychological domains are not wholly distinct in children's reasoning about biological processes which is in sharp contrast to previous studies that have demonstrated a demarcation between the psychological and biological domains (Kalish [3]; Notaro et al. [10]).

One interesting question that these results raise is why is there a distinct decrease by fourth grade in the recognition of the effects of psychobiological factors on biological processes. These results replicate the Raman and Gelman [7] findings in which there was a distinct drop in the entertainment of psychosocial factors after second grade. One possibility could be that with increasing socialization children recognize that psychobiological factors do not have an impact on biological processes such as growth. Second, the role of science education in elementary school might have a powerful role in the decrease of children thinking that psychological factors can affect biological processes.

There are a couple of potential criticisms of this study. First, although the "same" option is more parsimonious than the other two responses provided, participants who chose this response might be implying that psychobiological factors, in general (irrespective of whether they are "yummy" or "yucky"), affects growth. However, the justifications that were selected for the "same" responses across all age groups suggest that they thought that the quantity of the food for both characters in the vignettes was the same. This suggests that they were not entertaining psychobiological factors when choosing this option. Second, preschoolers and second graders might just be responding to the positive valence of the options in the questions. For example, they might be choosing the "yummy" response because this option is a term that they are familiar with and one that sounds good. Study 2 directly addresses this potential issue by presenting young children with vignettes that do not use the terms "yummy" and "yucky" but still taps into their understanding of psychobiological causal factors for the process of growth.

\section{Study 2}

Recall that one of the potential drawbacks of Study 1 was the fact that preschoolers and second graders could have been responding to the positive valence in the question, thus selecting the response that "yummy" foods promote 
growth. In order to control for this possibility, participants in this study were presented with vignettes that described a subset of the foods used in Study 1 without the use of the terms "yummy" and "yucky." Instead, the terms "taste really good" and "taste really bad" were substituted for the terms "yummy" and "yucky." Also, the vignettes in Study 2 involved three characters each one endorsing a different point of view about eating foods that taste really good or really bad, thus presenting all possible outcomes of eating foods that taste really good or really bad. Thus, if participants still endorse the notion that foods that "taste really good" promote growth, we can be confident that the young children in Study 1 were not just responding to the positive valence in the question.

3.1. Participants. Twelve preschoolers ( 7 females and 5 males, $M$ age $=4$ years 6 months, range $=4$ years to 4 years 10 months) were recruited for the study from preschools in a small Midwestern city.

Main Task. The main task presented each participant with two conditions, height and weight. Within each condition, each participant was presented with 6 vignettes ( 3 describing characters who consumed healthy foods and 3 describing characters who consumed unhealthy foods), resulting in each participant being presented with a total of 12 vignettes. The healthy foods that were presented to participants were peas, grapes, oatmeal, broccoli, cereal, and apples. The unhealthy foods were hash brown, jelly beans, French Fries, candy, donuts, and potato chips. The questions for the healthy and unhealthy foods were identical except for the foods listed in each vignette. An example for one of the vignettes for healthy foods is "Jake, Oliver, and Peter are having lunch together. Jake says "I think peas taste really good and you will grow tall if you eat really good tasting foods like peas." Oliver says "I think peas taste really bad and you will grow tall if you eat bad tasting foods like peas." Peter said "I don't think so, it doesn't matter if peas taste really good or really bad, you will grow tall if you eat peas because they have nutrients in them." Who do you agree with: (a) Jake; (b) Oliver; (c) Peter. To ensure that the preschoolers retained this information, three memory questions were asked. (a) Who thinks peas tastes really good? (b) Who thinks peas taste really bad? (c) Who thinks it does not matter how peas taste? Items were randomized within each condition and the response options and conditions were counterbalanced across participants such that half of the participants received the height condition first and the other half received the weight condition first.

3.2. Results. The coding of the data was " 1 " for the character who said that the food "tastes really good," " 0 " for the character who said it "tastes really bad," and " 0.5 " for the character who said "it doesn't matter." Recall that the primary focus of Study 2 was to determine if the positive and negative valence in Study 1 was causing participants to choose those responses. A 3-taste (yummy; yucky; same) repeated measures ANOVA was conducted. The results indicated a main effect for taste, $F(2,22)=9.96, P<.0006$.
Post hoc tests revealed that participants selected the "tastes really good" responses significantly more times than either of the "tastes really bad" or "the taste doesn't matter" responses, $P s<.01$ ( $M s=7.6,1.0$, and 3.3 for the "tastes really good," "tastes really bad," or "taste doesn't matter" responses).

3.3. Discussion. The objective of this study was to determine if the young children in Study 1 were merely responding to the positive valence in the question rather than attributing psychobiological causes to the process of growth. The results of Study 2 confirm that children are not just responding to the term "yummy" in the question since they respond in a similar manner to the vignettes in Study 2 even though the terms "yummy" and "yucky" are not present. Moreover, this study presents the biological counterargument that good taste or bad taste does not matter, so participants were presented with all possible options of the argument. Thus, the responses in Study 1 cannot be attributed to the valence of the terms in the vignettes but rather seem to support the notion that preschoolers and second graders entertain psychobiological causes for the process of growth.

\section{Study 3}

The results of Studies 1 and 2 suggest that young children subscribe to the notion that psychobiological factors affect height and weight. Based on these results, one might conclude that the psychological and biological domains are indistinguishable in the early preschool years (see also Carey [21]). In contrast, however, there is an extensive literature documenting early differentiation of the two domains (Opfer [22]). This raises the question as to whether children's endorsement of psychobiological factors is process specific or process general. Thus, they might endorse psychobiological causes for growth but not for other biological processes such as illness. To test this hypothesis, the same "yummy" and "yucky" healthy and unhealthy foods that were used in Study 1 were used to examine the effect that these labels had on the contraction of common contagious illnesses.

The biological process of contagious illness is an interesting phenomenon since the causal underpinnings are not visible. Also, unlike growth, the effect of nutrition on contracting an illness is indirect, since there needs to be the presence of contaminants such as germs for the contraction of an illness (i.e., nutrition by itself is not going to cause a person to contract a contagious illness). Moreover, unhealthy nutrition affects the resistance of the individual which in turn makes one more susceptible to the contraction of an illness. Thus, unlike growth, for children to recognize that nutrition affects the susceptibility to illness, there is an indirect causal link that they must recognize.

There are three distinct patterns of results, one of which we might expect. First, children may maintain a clear domain distinction and not entertain psychobiological labels as affecting the contraction of an illness. This is consistent with the results of previous research which supported the domain independence model (Notaro et al. [10, 20]; Opfer [22]). Second, children might recognize that yummy/yucky nutrition 
affects the contraction of illness (Raman and Gelman, [7]; Schulz [9]), like they did in Study 1. Finally, since the impact of nutrition on contracting a contagious illness is indirect, adults but not children might recognize that yummy/yucky nutrition can affect the susceptibility to contract an illness (Nemeroff [23]).

\subsection{Method}

4.1.1. Participants. The sample for this study included 25 preschoolers (11 girls and 14 boys, $M$ age $=4$ years 11 months, range $=4$ years 5 months to 5 years 2 months), 27 second graders ( 12 girls and 15 boys, $M$ age $=7$ years 5 months, range $=7$ years 2 months to 8 years 9 months), 22 fourth graders $(11$ girls and 11 boys, $M$ age $=9$ years 5 months, range $=9$ years 3 months to 10 years 6 months), and 31 adults ( 24 women and 7 men, $M$ age $=18$ years 5 months, range $=18$ years 2 months to 22 years 1 month). The participants were primarily European American and were from middle-income homes. None of the participants of Study 1 participated in this study.

Participants were residents of a small midwestern city. Children were recruited from preschools in the area. Adults were recruited from the introductory psychology pool and were given course credit for their participation.

4.1.2. Materials. Participants were presented with the same 6 healthy and 6 unhealthy foods used in Study 1. The difference here was that the vignettes asked if the character would contract a contagious illness instead of asking if the character would grow tall or fat. Each illness was presented twice, once for a healthy food and once for an unhealthy food. The illness items are ones that preschoolers are familiar with and they have been successfully used in other studies (Raman and Gelman $[4,7])$.

An example of a vignette that presented a healthy food was the following. Let me tell you some things about these three boys, Mark, Jake, and Oliver. Both Jake and Oliver eat the same amount of peas for dinner. Jake thinks that peas are a "yummy" food but Oliver thinks that peas are a "yucky" food. Did Oliver eat all his peas? (a) yes; (b) no. Did Jake at all his peas? (a) yes; (b) no. These two memory questions were asked to ensure that the participants retained the information provided in the vignettes and only participants who answered both of these questions correctly were included in the study. Now, remember that Jake thinks that peas are a "yummy" food but Oliver thinks that peas are a "yucky" food. Mark, Jake, and Oliver are playing together. Mark has a bad cough. Mark coughs all over Jake and Oliver. Who is more likely to get a cough? (a) Oliver; (b) Jake; (c) their chances of getting a cough are the same. If participants chose either option (a) or (b), they were asked "Why do you think (child's response) is more likely to get a cough? Is it because: (a) peas which are a (yummy/yucky depending on the child's response) food gives you a cough (psychological justification); (b) there are no nutrients in yummy/yucky foods so that will give you a cough (psychobiological justification); (c) there are no nutrients in the peas so they give you a cough (biological justification). Is there any other reason (child's response) got a cough?" If participants chose the "same" response, they were asked "Why are their chances of getting a bad cough the same?" Unlike Study 1, participants in this study were asked to generate justifications for all the vignettes since there were only 12 vignettes (as opposed to the 24 vignettes in Study 1).

4.1.3. Procedure. The procedure was identical to that of Study 1. Line drawings of the characters' faces were used to hold the attention of the child. The symptoms were not shown in any of the pictures, and the faces of the characters displayed neutral emotional expressions. The experimenter read the vignettes to the children, pointing to the relevant card when reading the item, and wrote down the children's responses. Participants received a total of 12 vignettes, 6 vignettes presented healthy foods and 6 vignettes presented unhealthy foods in a random order. College students received a booklet that contained the 12 vignettes (with no pictures) and were asked to write down their responses in a booklet. The presentation of the two characters in the response option was counterbalanced across participants, and the order of the justifications that were presented was randomized.

\subsection{Results}

Coding of the Judgments. The coding of the judgments and judgments and justifications was identical to Study 1. Each participant had two total scores for each of the categories of "yummy," "yucky," and "same" responses, one for healthy foods and one for unhealthy foods. A descriptive analyses of the data revealed that the majority of participants selected the "same" response for both healthy and unhealthy foods across all categories, thus the analyses will focus exclusively on the "same" response category (see Tables 3(a), 3(b), and 3(c) for means across age groups and conditions).

Judgments Analyses. A 2(food) $\times 4$ (age group) repeated measures ANOVA was conducted for the "same" responses. The results revealed nonsignificant results for both foods, $F(1,100)=0.38, P>.6$ and a non-significant food $\times$ age group interaction, $F(4,100)=.68, P>.5$. Across all age groups, participants overwhelmingly selected the "same" responses.

Analyses of the Justifications. Participants who chose the "same" response were then asked the open-ended question, "why do you think the chances of them getting (the illness in question) is the same?" Two coders independently coded the open-ended responses. The interrater reliability was 1.0. The coding of these judgments and justifications revealed that $100 \%$ of the participants across all age groups generated biological justifications. An example of one such justification from a preschooler was "germs cause illness not food."

4.3. Discussion. The results of this study revealed that even young children do not subscribe to psychobiological labels when assessing the impact of "yummy" and "yucky" 
TABle 3: (a) Mean number of "yummy" responses (out of a maximum of 6). (b) Mean number of "yucky" responses (out of a maximum of 6). (c) Mean number of "same" responses (out of a maximum of 6).

(a)

\begin{tabular}{lcc}
\hline Age group & Healthy & Unhealthy \\
\hline Preschool & 0.0 & 0.0 \\
Second & 0.3 & 0.1 \\
Fourth & 0.1 & 0.1 \\
Adults & 0.1 & 0.0 \\
\hline
\end{tabular}

(b)

\begin{tabular}{lcc}
\hline Age group & Healthy & Unhealthy \\
\hline Preschool & 0.0 & 0.0 \\
Second & 0.3 & 0.1 \\
Fourth & 0.1 & 0.0 \\
Adults & 0.1 & 0.0 \\
\hline
\end{tabular}

(c)

\begin{tabular}{lcc}
\hline Age group & Healthy & Unhealthy \\
\hline Preschool & 5.8 & 5.7 \\
Second & 5.6 & 5.4 \\
Fourth & 5.9 & 5.9 \\
Adults & 6.0 & 6.0 \\
\hline
\end{tabular}

nutrition on common contagious illnesses. This is in sharp contrast to Study 1 which generated evidence of young children entertaining the notion that the affective quality of the food will have an effect on growth. The results of Study 3 support the argument that children do not entertain nonbiological causes for the origins of illness (Notaro [10, 20]; Raman and Gelman [4]).

An interesting question that emerges from the results of Study 2 is why children endorse psychobiological causes for the biological process of growth but not for illness. There are at least two hypotheses as to why this might be the case. First, the association of food as a causal factor for contagious illnesses is indirect. The quality of nutrition does not directly cause illness, but biological contaminants such as germs or viruses are responsible for contagious illness. The nutritive value of food is responsible for the overall health of the individual which in turn will dictate if the person contracts a contagious illness when he/she comes in contact with viruses and germs. Thus, when two people are exposed to the same germs or viruses, one person may get sick while the other person may not get sick, depending on the physical health of each individual. With the biological process of growth, however, the quality of nutrition has a more direct effect on the height and weight of an individual so it might have been easier for children to associate psychobiological causes with the process of growth but not illness. Second, young children may not be interpreting the terms "yummy" and "yucky" as having a purely psychological connotation which in turn might be responsible for the difference in results when young children reason about the impact on growth and illness.
A surprising result of Study 3 is that even adults did not associate nutrition with the chances of contracting an illness, but like preschoolers stated that germs cause illness. Taken together, these results indicate that children and adults expect different effects of psychobiological factors for different biological processes. Correspondingly, these results support the interpretation that even when children expect psychobiological factors to affect biological processes, they differentiate the effects between the biological processes.

Similar to Study 1, one potential criticism of the study is that the "same" option could suggest that both "yummy" and "yucky" foods are responsible for contracting an illness, thus both characters have the same chance of contracting an illness. However, participants' justifications revealed that they were not endorsing both psychobiological factors as all the participants generated biological justifications for why they chose the "same" response.

\section{General Discussion}

The three studies reported in this paper examined if children and adults entertain psychobiological labels when reasoning about the biological processes of growth and illness. The studies are the first to examine the impact and the underlying mechanisms that children and adults use when reasoning about the impact of psychobiological factors on growth and illness. From a biological perspective, psychological causes such as the "yumminess" or the "yuckiness" of a food should not have an effect on growth or whether one gets sick as long as both people eat the same quantity of the food in question. More broadly, affective factors are psychological at their core and thus should not play a role in a biological process such as growth or the transmission of contagious illnesses.

Three patterns of outcomes were possible in the current set of studies. The first possibility is that psychobiological factors would not have an effect on biological processes such as growth and illness. This result would be consistent with the literature demonstrating domain differentiation by preschool age (Notaro et al. [10]). The second possibility is that children and adults might show a psychologicalbiological domain interaction for growth and/or illness (Nemeroff and Rozin [24]; Raman and Gelman [7]). This would support the domain-interaction model.

In the present set of studies, young children (preschoolers and second graders) selectively applied psychobiological labels such as the "yumminess" of the food as affecting growth (height and weight) but not illness. Unlike older children and adults, the youngest children (preschoolers and second graders) reasoned that if a healthy food tasted "yummy," it would have more of an effect on height than a "yucky" healthy food. Similarly, an unhealthy "yummy" food would have more of an effect on weight than a healthy "yummy" food. Across most age groups, when participants selected psychobiological responses, the justifications they selected were purely psychological. These results support the grade trend that Raman and Gelman [7] found with psychosocial factors where preschoolers and second graders reasoned that any kind of association would reduce 
the chances of contracting a contagious illness. This result is consistent with the notion that children entertain crossdomain boundaries on this task.

However, a surprising result is that young children did not entertain psychobiological causes for the biological process of illness. Instead, across all age groups, participants justified their responses by stating that both characters have the same chances of getting sick and that germs or microorganisms cause illness, not the "yumminess" or the "yuckiness" of a food. These results corroborate the results of studies that have demonstrated that young children treat the biological and psychological domains as wholly distinct even in realm of illness (Notaro et al. [15]). This might seem surprising in the light of previous studies that have demonstrated that young children and even adults think that the psychological and biological domains interact (Nemeroff [23]; Schultz et al. [9]; Raman and Gelman [7]) when reasoning about the causes of contagious illness.

One of the pressing questions is why do children apply psychobiological causes to growth but not to illness? One possibility is that nutrition does not directly cause the contraction of an illness like it does for height and weight. Thus, when reasoning about illness, children are presented with a more challenging situation than when they are presented with the impact of nutrition on growth. Unlike previous studies, these three studies suggest that children's appreciation of psychological and biological domains is process specific rather than process general, so we cannot make a global attribution that children will demonstrate the same type of reasoning for all biological processes.

The present results provide support for the suggestion that depending on the biological process, children entertain either subtle domain distinctions or cross-domain interactions. For example, they demonstrate domain interaction for the process of growth but demonstrate domain independence for the process of illness. An important issue is whether we can predict when children will appropriately or inappropriately predict that domains will interact or be independent. One possibility is that when a direct causal factor that affects a biological process (nutrition directly affects growth but not illness for which the intermediate factor of germs has to be there to contract a contagious illness), children might demonstrate more of a domain interaction than domain independence form of reasoning.

There are a few limitations to these studies. First, it can be argued that the variety of healthy and unhealthy foods presented in these studies are not completely all healthy or unhealthy. For example, cereal can have high amounts of sugar in it which one might argue is unhealthy. Similarly, French Fries and hash browns do have nutrients like potassium and dietary fiber both of them which are considered to be beneficial. Overall though, the healthy foods presented in these studies are not fried and are relatively high in nutritional value whereas the unhealthy foods are all relatively high in fat (as assessed by children and adults themselves).

Second, we had to limit the number of healthy and unhealthy foods that were presented so that the task would not be too cognitively demanding for preschoolers. It is important to recognize that these studies are a first step in investigating children's understanding of the impact of psychobiological labels on biological processes such as growth and illness and that these studies do not provide a comprehensive understanding to children's reasoning about the effects of psychobiological factors on all biological processes.

Third, participants in both studies were primarily middle-class Euro-Americans, limiting the generalizability of these results to other ethnicities and socioeconomic groups. It would be worthwhile to conduct these studies with minority and low socioeconomic groups to determine if they have similar conceptual ideas about the effects of eating healthy and unhealthy foods.

There are several directions that future research can take.linebreak First, given the prevalence of childhood obesity, it is important to correct the misconception that a "yummy" tasting food actually promotes growth, since with most foods, "yummy" tasting foods are actually unhealthy and do not promote healthy growth. Second, the misconception that nutrition does not impact the contraction of illness for both children and adults should be corrected by educating children and adults how healthy a person is will make a difference in whether one contracts a contagious illness or not. Third, it would be beneficial to examine the effects of psychobiological causes on other biological processes such as digestion to determine if children think that affective labels such as "yummy" and "yucky" foods might impact this process. Fourth, investigating from where and what kinds of input children get about nutrition and how children make the distinction between healthy and unhealthy foods is an important avenue for future research. Nguyen [25] found that 3- and 4-year olds preferred external input from a mother or teacher as opposed to artifacts and other adults when it came to assessing if a food was "yummy" or "yucky." Finally, in light of the increasing rates of childhood obesity, it would be interesting to determine at what age children recognize that too much weight gain can be harmful to an individual's well-being which in turn can make the individual more susceptible to illness.

Overall, these studies are a first step in demonstrating the important developmental changes that are taking place between preschool and middle childhood in how children come to understand the impact of psychobiological factors on biological processes. The results demonstrate that children's causal theories are process specific which lends an alternate explanation to the long-standing debate as to if and when children entertain an autonomous theory of biology.

\section{Acknowledgments}

Preparation of this paper was supported by a grant from the National Institute of Child Health and Human Development 1 R15 HD058303-01A1 to Raman. The author would like to thank the parents, children, and teachers in the Oxford School district for their participation. He also thank Jennifer Kelley and Alessa Petersen for their assistance in data collection. I am grateful to Todd Shackelford for his constructive comments and feedback on a draft of the paper. 


\section{References}

[1] A. G. Backscheider, M. Shatz, and S. A. Gelman, "Preschoolers ability to distinguish living kinds as a function of regrowth," Child Development, vol. 64, no. 4, pp. 1242-1257, 1993.

[2] K. Inagaki, "Emerging distinction between naïve biology and naïve psychology," in The Emergence of Core Domains of Thought: Children's Reasoning about Physical, Psychological, and Biological Phenomena, H. M. Wellman and K. Inagaki, Eds., pp. 27-44, Jossey-Bass, San Francisco, Calif, USA, 1997.

[3] C. W. Kalish, "Preschoolers' understanding of mental and bodily reactions to contamination: what you don't know can hurt you, but cannot sadden you," Developmental Psychology, vol. 33, no. 1, pp. 79-91, 1997.

[4] L. Raman and S. A. Gelman, "Children's understanding of the transmission of genetic disorders and contagious illnesses," Developmental Psychology, vol. 41, no. 1, pp. 171-182, 2005.

[5] K. Springer and F. C. Keil, "Early differentiation of causal mechanisms appropriate to biological and nonbiological kinds," Child Development, vol. 62, no. 4, pp. 767-781, 1991.

[6] K. Springer and J. Ruckel, "Early beliefs about the cause of illness: evidence against immanent justice," Cognitive Development, vol. 7, no. 4, pp. 429-443, 1992.

[7] L. Raman and S. A. Gelman, "Do children endorse psychosocial factors in the transmission of illness and disgust?" Developmental Psychology, vol. 44, no. 3, pp. 801-813, 2008.

[8] L. Raman, "Can we get sick if we want to? Children's and adults' recognition of intentionality in the origins of illness and injuries," British Journal of Psychology, vol. 100, no. 4, pp. 721-751, 2009.

[9] L. E. Schulz, E. B. Bonawitz, and T. Griffiths, "Can being scared cause tummy aches? Naïve theories, ambiguous evidence, and preschoolers' causal inferences," Developmental Psychology, vol. 43, no. 5, pp. 1124-1139, 2007.

[10] P. C. Notaro, S. A. Gelman, and M. A. Zimmerman, "Children's understanding of psychogenic bodily reactions," Child Development, vol. 72, no. 2, pp. 444-459, 2001.

[11] K. Inagaki, "Emerging distinction between naïve biology and naïve psychology," New Directions for Child Development, vol. 75, pp. 27-44, 1997.

[12] K. Inagaki and G. Hatano, "Young children's understanding of the mind-body distinction," Child Development, vol. 64, no. 5, pp. 1534-1549, 1993.

[13] H. M. Wellman and C. N. Johnson, "Children's understanding of food and its functions: a preliminary study of the development of concepts of nutrition," Journal of Applied Developmental Psychology, vol. 3, no. 2, pp. 135-148, 1986.

[14] I. Contento, "Children's thinking about food and eating: a Piagetian-based study," Journal of Nutrition Education, vol. 13, pp. 86-90, 1981.

[15] S. Nguyen, "Children's evaluative categories and inductive inferences within the domain of food," Infant and Child Development, vol. 17, no. 3, pp. 285-299, 2008.

[16] V. Slaughter and C. Ting, "Development of ideas about food and nutrition from preschool to university," Appetite, vol. 55, no. 3, pp. 556-564, 2010.

[17] M. C. McKinley, C. Lowis, P. J. Robson et al., "It's good to talk: children's views on food and nutrition," European Journal of Clinical Nutrition, vol. 59, no. 4, pp. 542-551, 2005.

[18] H. Guerin and J. P. Thibaut, "Les développments des représentations sur les ailments chez l'enfant de 4 à 12 ans," Enfance, vol. 60, pp. 251-260, 2008.

[19] L. Raman, Children's and Adults' Understanding of Nutrition on Growth and Mood States, Poster Presented at the Cognitive Development Society, San Antonio, Tex, USA, 2009.
[20] P. C. Notaro, S. A. Gelman, and M. A. Zimmerman, "Biases in reasoning about the consequences of psychogenic bodily reactions: domain boundaries in cognitive development," Merrill-Palmer Quarterly, vol. 48, no. 4, pp. 427-449, 2002.

[21] S. Carey, Conceptual Change in Childhood, MIT Press, Cambridge, Mass, USA, 1985.

[22] J. E. Opfer, "Identifying living and sentient kinds from dynamic information: the case of goal-directed versus aimless autonomous movement in conceptual change," Cognition, vol. 86, no. 2, pp. 97-122, 2002.

[23] C. J. Nemeroff, "Magical thinking about illness virulence: conceptions of germs from "Safe" versus "Dangerous" others," Health Psychology, vol. 14, no. 2, pp. 147-151, 1995.

[24] C. Nemeroff and P. Rozin, "'You are what you eat': applying the demand-free "impressions" technique to an unacknowledged belief," Ethos, vol. 17, pp. 50-69, 1989.

[25] S. Nguyen, "The role of external sourcesof information on children's evaluative food categories," Infant and Child Development. In press. 


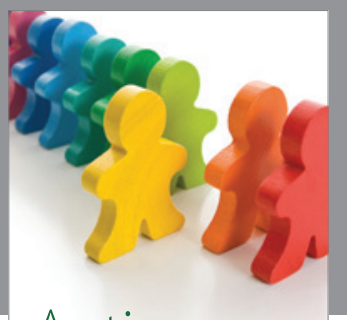

Autism

Research and Treatment
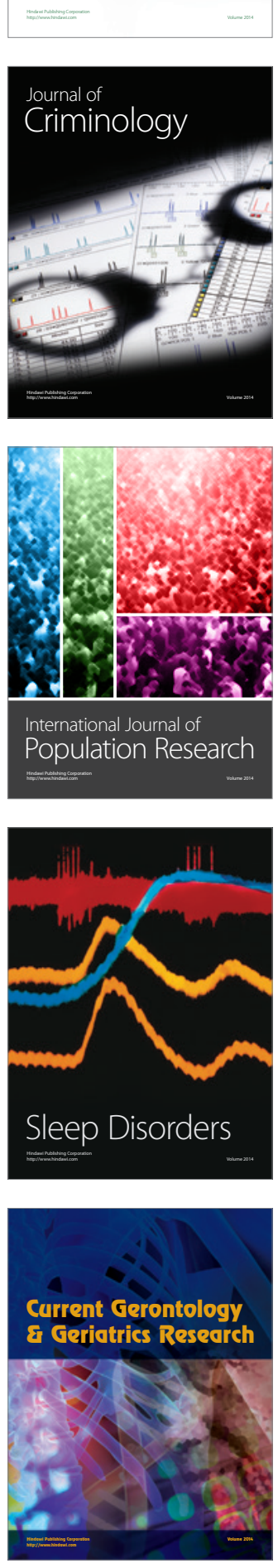
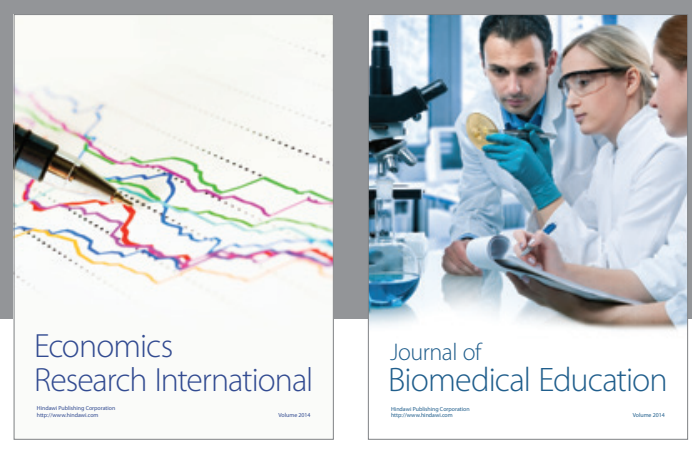

Journal of

Biomedical Education

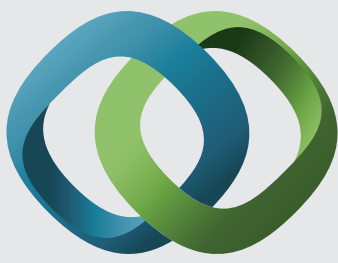

\section{Hindawi}

Submit your manuscripts at

http://www.hindawi.com
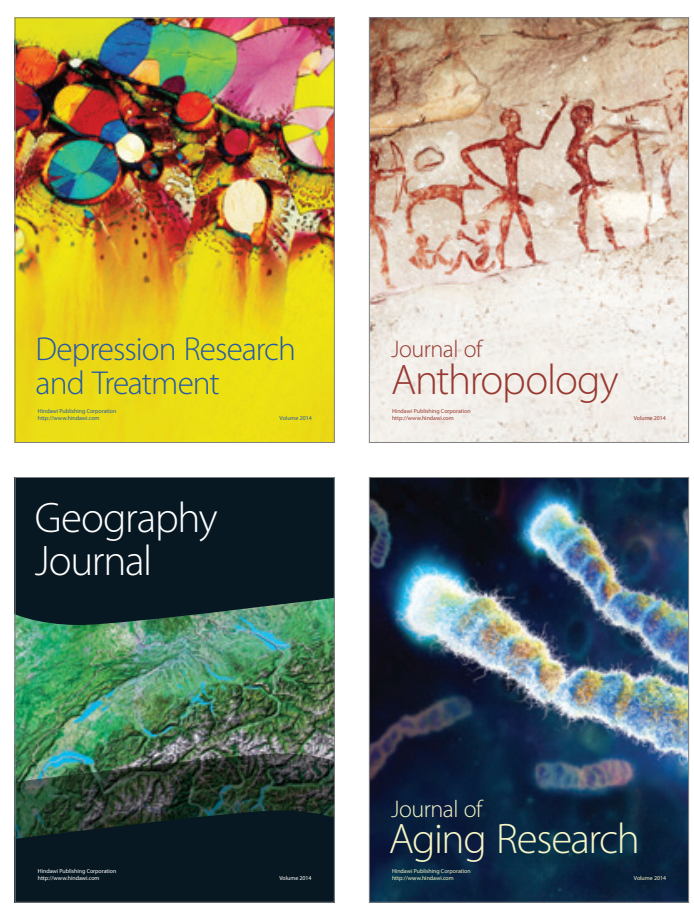

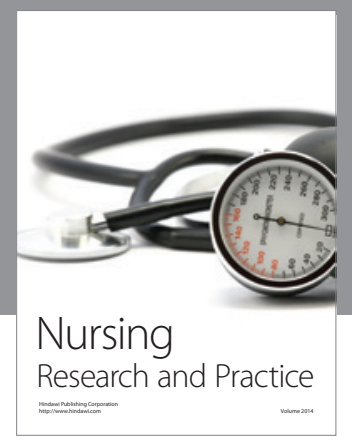

Nursing

Research and Practice

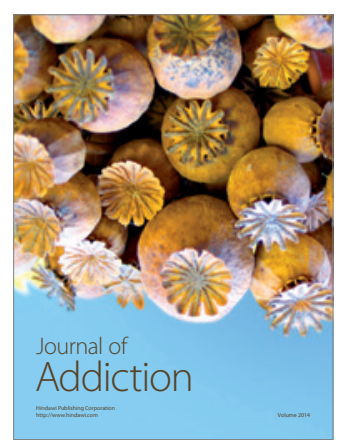

Child Development

Research

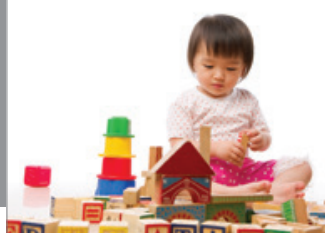

迥
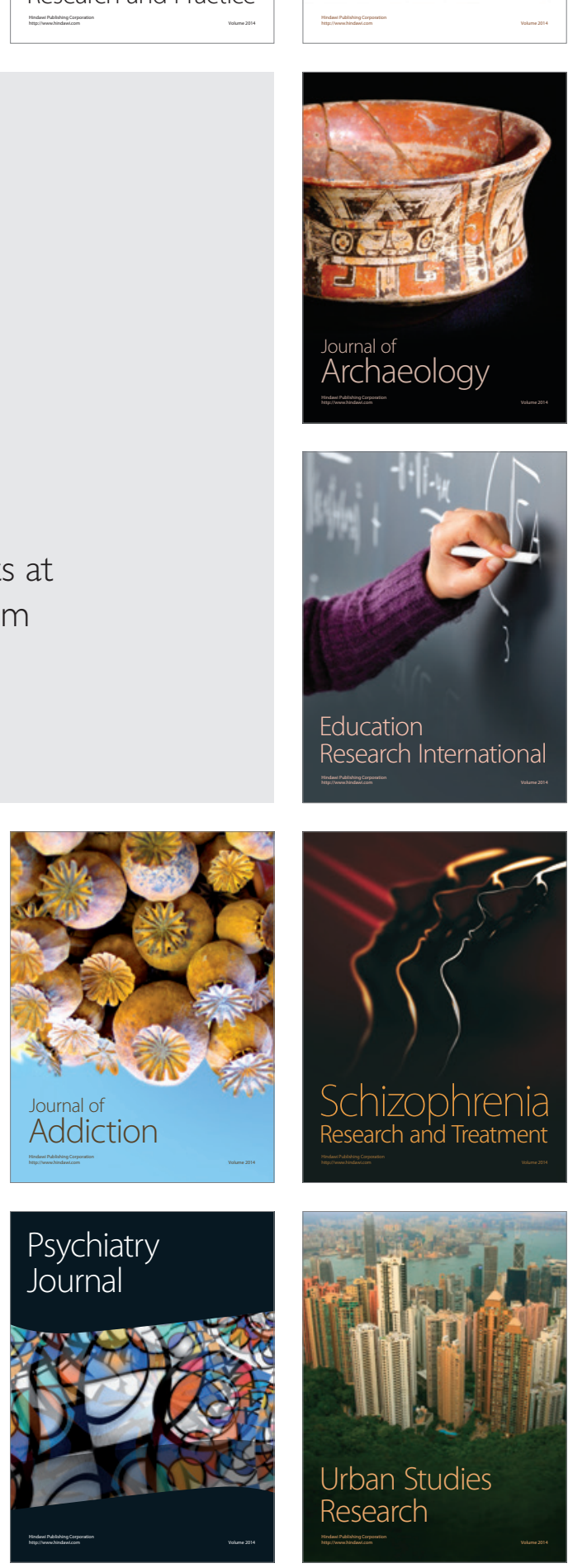